\title{
All vertex-transitive locally-quasiprimitive graphs have a semiregular automorphism
}

\author{
Michael Giudici · Jing Xu
}

Received: 13 October 2005 / Accepted: 18 July 2006 /

Published online: 22 August 2006

(C) Springer Science + Business Media, LLC 2007

\begin{abstract}
The polycirculant conjecture states that every transitive 2-closed permutation group of degree at least two contains a nonidentity semiregular element, that is, a nontrivial permutation whose cycles all have the same length. This would imply that every vertex-transitive digraph with at least two vertices has a nonidentity semiregular automorphism. In this paper we make substantial progress on the polycirculant conjecture by proving that every vertex-transitive, locally-quasiprimitive graph has a nonidentity semiregular automorphism. The main ingredient of the proof is the determination of all biquasiprimitive permutation groups with no nontrivial semiregular elements.
\end{abstract}

Keywords Locally-quasiprimitive graphs $\cdot$ Semiregular automorphism · Biquasiprimitive $\cdot$ 2-Closure

\section{Introduction}

A semiregular permutation is a permutation whose cycles all have the same length. Such a permutation generates a semiregular permutation group, that is, a group such that the only element which fixes a point is the identity. Thus the existence of a nonidentity semiregular permutation in a permutation group is equivalent to the existence

M. Giudici is supported by an Australian Postdoctoral Fellowship.

J. Xu was supported by an IPRS scholarship of Australia.

M. Giudici · J. Xu $(\bowtie)$

The University of Western Australia, School of Mathematics and Statistics,

Crawley 6009, WA, Australia

M. Giudici

e-mail: giudici@maths.uwa.edu.au

J. Xu

Present address: School of Mathematical Sciences, Peking University, Beijing, 100871, P.R. China e-mail: xujing@math.pku.edu.cn. 
of a fixed point free element of prime order. A permutation group is regular if it is both transitive and semiregular. Moreover, a vertex-transitive graph has a regular group of automorphisms if and only if it is a Cayley graph. In 1981, Marušič [22] asked if every finite vertex-transitive digraph with at least two vertices has a nontrivial semiregular automorphism. This question was reproposed by Jordan [18] in 1988. It has been shown that every vertex-transitive graph of valency three [23] or four [7] has a nonidentity semiregular automorphism. Marušič [22] proved that all vertex-transitive digraphs with $p^{k}$ or $m p$ vertices, where $p$ is a prime and $m \leq p$, have a semiregular automorphism of order $p$. Also Marušič and Scapellato [23] proved that every vertextransitive digraph with $2 p^{2}$ vertices, for $p$ a prime, has a semiregular automorphism of order $p$.

In was proved in [12] that every transitive permutation group of degree at least 2 has a fixed point free element of prime power order. However, they also constructed transitive permutation groups with no fixed point free elements of prime order. Hence the existence of semiregular elements in automorphism groups of digraphs is not purely a problem about transitive permutation groups. We call a transitive permutation group elusive if it contains no fixed point free elements of prime order. As well as the examples in [12], the Mathieu group $M_{11}$ in its 3-transitive action on 12 points is elusive. See [2] and [14] for more examples.

The 2-closure $G^{(2)}$ of a permutation group $G$ is the largest subgroup of $\operatorname{Sym}(\Omega)$ with the same set of orbits on ordered pairs as $G$. The 2-closure of a 2-transitive group is the full symmetric group. On the other hand, we say that $G$ is 2 -closed if it is equal to its 2-closure. The full automorphism group of a digraph is 2-closed since any permutation of the vertex set which preserves the orbits of $\operatorname{Aut}(\Gamma)$ on ordered pairs preserves adjacency. However, not every 2-closed permutation group is the full automorphism group of some digraph. For example, an elementary abelian group of order 4 in its regular action on 4 points is 2-closed but is not the full automorphism group of any digraph. Klin [3] extended the question of Marušič to the more general setting of 2-closed groups. This leads to what is now known as the polycirculant conjecture (see [2]), that every finite transitive 2-closed permutation group of degree at least two contains a nontrivial semiregular permutation. In the original context of automorphism groups of digraphs, the name suggests that vertex-transitive digraphs contain a bunch of cycles and so have nice representations like the ones in [1]. It is a consequence of [13, Theorem 1.2] that if a counterexample $G$ exists then every minimal normal subgroup of $G$ is intransitive. Moreover, by [6, Theorem 4.1], the degree of any counterexample is divisible by a square.

In this paper we prove the truth of the polycirculant conjecture for the automorphism groups of a large class of graphs. A transitive permutation group $G$ on a set $\Omega$ is primitive if $G$ preserves no nontrivial partition of $\Omega$. If $G$ is primitive then every nontrivial normal subgroup of $G$ is transitive. This leads to the more general concept of a quasiprimitive permutation group which is one for which every nontrivial normal subgroup is transitive. All primitive groups are quasiprimitive, while not all quasiprimitive groups are primitive, for example, the action of any nonabelian simple group on the set of cosets of a nonmaximal subgroup. We say that a graph $\Gamma$ with a group $G$ of automorphisms is $G$-locally-quasiprimitive if for each vertex $\alpha$, the vertex stabiliser $G_{\alpha}$ acts quasiprimitively on the set $\Gamma(\alpha)$ of vertices adjacent to $\alpha$. Locally quasiprimitive graphs were studied in many papers, such as [20,25]. 
The class of all vertex-transitive locally-quasiprimitive graphs includes all vertextransitive 2-arc-transitive graphs. A graph is $s$-arc-transitive if the automorphism group acts transitively on the set of all $(s+1)$-tuples $\left(\alpha_{0}, \alpha_{1}, \alpha_{2}, \ldots, \alpha_{s}\right)$ such that $\alpha_{i}$ is adjacent to $\alpha_{i+1}$ and $\alpha_{i} \neq \alpha_{i+2}$. If a graph is vertex-transitive and $s$-arc-transitive with $s \geq 2$ then it is also 2-arc-transitive. Moreover, the stabiliser of a vertex acts 2-transitively on the set of neighbours of that vertex. The study of $s$-arc-transitive graphs goes back to Tutte $[32,33]$ who showed that graphs of valency 3 are at most 5-arc-transitive. Using the Classification of Finite Simple Groups, Weiss [34] showed that graphs of valency at least three are at most 7-arc-transitive. Praeger initiated a systematic study of 2-arc-transitive graphs [27] by showing that all nonbipartite examples are covers of 2-arc-transitive graphs where the automorphism group is quasiprimitive on vertices. This motivated the O'Nan-Scott Theorem for quasiprimitive permutation groups and has led to much work on both quasiprimitive 2-arc-transitive graphs $[10,11,15,17,21]$ and their covers [8, 9]. We also refer the reader to the surveys [28, 29]. Further work on 2-arc-transitive graphs has been done, for example, in [19, 24].

We prove the following theorem.

Theorem 1.1. Let $\Gamma$ be a finite graph with a group $G$ of automorphisms such that $G$ is vertex-transitive and locally-quasiprimitive. Then $\Gamma$ has a nonidentity semiregular automorphism.

Since all transitive groups of prime degree are primitive, we have the following corollary.

Corollary 1.2. Every finite arc-transitive graph of prime valency has a nonidentity semiregular automorphism. Also every finite 2-arc transitive, vertex-transitive graph has a nonidentity semiregular automorphism.

Theorem 1.1 also has the following analogue in the 2-closed setting.

Theorem 1.3. Let $G$ be a finite transitive permutation group on $\Omega$ and suppose that for $\omega \in \Omega$ there is a self paired orbit $\Sigma$ of $G_{\omega}$ on $\Omega \backslash\{\omega\}$ such that $G_{\omega}^{\Sigma}$ is quasiprimitive. Then $G^{(2)}$ contains a nontrivial semiregular permutation.

It was shown in [25], that if $\Gamma$ is a $G$-locally quasiprimitive graph with $G$-vertextransitive and $N$ a normal subgroup of $G$, then either $N$ has at most 2 orbits on vertices or $N$ is semiregular. Thus if $G$ does not contain a nontrivial semiregular element then $G$ is either quasiprimitive or biquasiprimitive. A biquasiprimitive permutation group is a transitive permutation group for which every nontrivial normal subgroup has at most two orbits and there is some normal subgroup with precisely two orbits. This is an important class of permutation groups when studying bipartite graphs (see for example $[16,17,26])$ and were studied in their own right in [30]. All quasiprimitive elusive groups were determined in [13] and shown to be of the form $M_{11} 2 K$ acting on $12^{k}$ points, for some transitive subgroup $K$ of $S_{k}$. Moreover, such groups are not 2-closed. In this paper we determine all biquasiprimitive elusive groups and this enables us to 
prove Theorem 1.1. Note that we use $[A: B]$ to denote the set of right cosets of a subgroup $B$ in a group $A$ and use $|A: B|$ for the index of $B$ in $A$.

Theorem 1.4. Let $G$ be a finite biquasiprimitive elusive permutation group on $\Omega$ and let $\alpha \in \Omega$. Then one of the following holds:

1. $G=M_{10}$ and $|\Omega|=12$;

2. $G=M_{11}^{k} \rtimes K \leqslant M_{11} 2 S_{k}$ and $G_{\alpha} \cong \operatorname{PSL}(2,11)^{k} \rtimes K^{\prime}$, where $k$ is a positive integer, $K^{\prime} \leqslant K \leqslant S_{k}$ such that $K$ is transitive, $\left|K: K^{\prime}\right|=2$, and $K \backslash K^{\prime}$ contains no elements of order 2 ;

3. $G=M_{11}^{k} \rtimes K \leqslant M_{11} 2 S_{k}$ and $G_{\alpha} \cong\left(\operatorname{PSL}(2,11)^{k / 2} \times M_{11}^{k / 2}\right) \rtimes K^{\prime}$, where $k$ is an even positive integer, $K^{\prime} \leqslant K \leqslant S_{k}$ such that $K$ is transitive and $K^{\prime}$ is intransitive, $\left|K: K^{\prime}\right|=2$ and $K \backslash K^{\prime}$ contains no elements of order 2 .

Moreover, each group $G$ in (1)-(3) is biquasiprimitive and elusive, $G$ is not 2-closed, and $G^{(2)}$ contains a fixed point free element of order 3.

We note that for the examples in Theorem 1.4 (2), the unique minimal normal subgroup $N=M_{11}^{k}$ of $G$ acts faithfully on both its orbits, while in Theorem 1.4 (3) the unique minimal normal subgroup $N=M_{11}^{k}$ of $G$ is unfaithful on each of its orbits.

This paper is set out as follows. We first present some preliminary results we need in Section 2. In Section 3 we give several constructions of biquasiprimitive elusive groups and then in Section 4 show that these are the only examples, thus completing the proof of Theorem 1.4. Finally, in Section 5 we prove Theorems 1.1 and 1.3.

\section{Preliminary results}

In this section we collect some results which will be needed throughout the paper.

Lemma 2.1 ([23, Lemma 2.1]). Let $G$ be an elusive permutation group with point stabiliser $G_{\alpha}$. Then every conjugacy class of elements of prime order in $G$ intersects $G_{\alpha}$ non-trivially.

We have the following important example.

Example 2.2 (see [2]). The Mathieu group $M_{11}$ in its 3-transitive action on 12 points with point stabiliser $\operatorname{PSL}(2,11)$ is elusive since it has only one conjugacy class of elements of order 2 or 3 . Moreover, since $M_{11}$ is 2 -transitive we have that $M_{11}^{(2)}=S_{12}$.

Since $M_{10}=A_{6} \cdot 2$ (with point stabiliser $A_{5}$ ) is a transitive subgroup of the above permutation group $M_{11}$, it is also elusive. The socle $T=A_{6}$ has two orbits of length 6 and so $M_{10}$ is biquasiprimitive. Moreover, given $\alpha$ in one $T$-orbit, $T_{\alpha}$ is transitive on the other $T$-orbit. Hence Wielandt's Dissection Theorem ([35, Theorem 6.5], see also [2, Theorem 5.5]) implies that $T^{(2)}$ contains $A_{6} \times A_{6}$, and so $T^{(2)}$ contains many fixed point free elements of order 3 . Therefore, $M_{10}^{(2)}$ is not elusive.

We can use these examples to build many more examples of elusive groups. 
Proposition 2.3 ([2, Theorem 4.1]). Let $G_{1} \leqslant \operatorname{Sym}\left(\Omega_{1}\right)$ be an elusive permutation group on $\Omega_{1}$ and let $G_{2} \leqslant \operatorname{Sym}\left(\Omega_{2}\right)$ be an elusive permutation group on $\Omega_{2}$. Then

1. $G_{1} \times G_{2}$ acts elusively on $\Omega_{1} \times \Omega_{2}$; and

2. $G_{1} 2 S_{k}$ is elusive on $\Omega_{1}^{k}$ in the natural product action.

Corollary 2.4. Let $\Omega=\Delta^{k}$ for some $k \geq 1$ and $|\Delta|=12$. Then both $M_{11}^{k}$ and $M_{11} 2$ $K$, where $K \leqslant S_{k}$, are elusive permutation groups on $\Omega$.

In fact, we have just constructed all elusive permutation groups with a transitive minimal normal subgroup, and hence all quasiprimitive elusive groups.

Theorem 2.5 ([13, Theorem 1.1]). Let $G$ be an elusive permutation group on a finite set $\Omega$ which has at least one transitive minimal normal subgroup. Then $G=M_{11} 2 K$ acting with its product action on $\Omega=\Delta^{k}$ for some $k \geq 1$, where $K$ is a transitive subgroup of $S_{k}$ and $|\Delta|=12$.

We will also need the following two results.

Proposition 2.6 ([13, Proposition 2.1]). Let $N=T^{k}$, where $T$ is a finite nonabelian simple group, and suppose that $N$ acts elusively on $\Omega$. Then $T=M_{11}$ and the action of $N$ on $\Omega$ is the product action on $\Delta^{k}$ where $|\Delta|=12$.

Theorem 2.7 ([13, Theorem 1.3]). Let $T$ be a simple group with a proper subgroup $H$ which meets every Aut $(T)$-conjugacy class of elements of $T$ of prime order. Then $T$ is one of $A_{6}, M_{11}, \mathrm{P} \Omega^{+}(8,2)$ or $\mathrm{P} \Omega^{+}(8,3)$, and $H=A_{5}, \operatorname{PSL}(2,11), \mathrm{P} \Omega(7,2)$ or $\mathrm{P} \Omega(7,3)$, respectively. Furthermore, if $H$ meets every conjugacy class of elements of $T$ of prime order, then $T=M_{11}$ and $H=\operatorname{PSL}(2,11)$.

We also need a couple of results about 2-closures.

Lemma 2.8 ([35, Theorem 5.7]). Let $G$ and $L$ be permutation groups on a set $\Omega$. If $G \leqslant L$, then $G^{(2)} \leqslant L^{(2)}$.

\section{Proposition 2.9.}

1. [2, Theorem 5.1] Let $G_{1} \leqslant \operatorname{Sym}\left(\Omega_{1}\right)$ and $G_{2} \leqslant \operatorname{Sym}\left(\Omega_{2}\right)$ be transitive permutation groups. Then in the action of $G_{1} \times G_{2}$ on $\Omega_{1} \times \Omega_{2}$, given by $(\alpha, \beta)^{\left(g_{1}, g_{2}\right)}=$ $\left(\alpha^{g_{1}}, \beta^{g_{2}}\right)$, we have $\left(G_{1} \times G_{2}\right)^{(2)}=G_{1}^{(2)} \times G_{2}^{(2)}$.

2. [31, Proposition 3.1] Let $G \leqslant \operatorname{Sym}(\Omega)$ and $K \leqslant S_{k}$. Then in the product action on $\Omega^{k}$ we have that $G^{(2)}$ ? $K \leqslant(G>K)^{(2)}$.

The next theorem is very important for our proof of Theorem 1.1, see also [20, Theorem 1.3].

Theorem 2.10 ([25, Section 1]). Let $\Gamma$ be a finite connected G-vertex-transitive, $G$-locally quasiprimitive graph, and let $N$ be a normal subgroup of $G$. Then one of the following holds. 
1. $N$ is transitive on the vertex set $V \Gamma$; or

2. $\Gamma$ is bipartite and the $N$-orbits in $V \Gamma$ are the two parts of the bipartition of $\Gamma$; or

3. $N$ has more than two orbits in $V \Gamma$, and $N$ is semiregular on $V \Gamma$.

We also need the following well known lemma from permutation group theory. See for example [5, Theorem 4.2A].

Lemma 2.11 ([5, Theorem 4.2A]). Let $G$ be a transitive subgroup of $\operatorname{Sym}(\Omega)$, and $\alpha$ a point in $\Omega$. Let $C$ be the centraliser of $G$ in $\operatorname{Sym}(\Omega)$. Then $C$ is semiregular, and $C \cong N_{G}\left(G_{\alpha}\right) / G_{\alpha}$.

\section{Some biquasiprimitive elusive groups}

\subsection{The notation for biquasiprimitive elusive groups}

Throughout this paper, all groups and graphs are finite. We adopt the notation used in [30] for biquasiprimitive permutation groups. Suppose that $G$ is a biquasiprimitive elusive permutation group on a finite set $\Omega$. Then there exists a non-trivial intransitive normal subgroup of $G$ which has two orbits, say $\Delta_{1}, \Delta_{2}$. Thus there is a set $\Delta$ such that we can identify $\Omega$ with $\Delta \times\{1,2\}$ such that $\Delta_{1}=\Delta \times\{1\}$ and $\Delta_{2}=\Delta \times\{2\}$. Each element of $G$ either fixes the two orbits setwise or interchanges them. The elements of $G$ which fix $\Delta_{1}$ and $\Delta_{2}$ setwise form a subgroup $G^{+}$of index 2 in $G$, and $G^{+}$induces a transitive permutation group $H$ on $\Delta$. By the embedding theorem for permutation groups, $G$ is conjugate in $\operatorname{Sym}(\Omega)$ to a subgroup of the wreath product $H$ ₹ $S_{2}=(H \times H) \rtimes S_{2}$, where for $\left(y_{1}, y_{2}\right)$ in the base group $H \times H$, and (12) $\in S_{2}$,

$$
(\delta, i)^{\left(y_{1}, y_{2}\right)}=\left(\delta^{y_{i}}, i\right) \quad \text { and } \quad(\delta, i)^{(12)}=\left(\delta, i^{(12)}\right) \quad \text { for all }(\delta, i) \in \Omega
$$

We write the base group $B=H \times H$ as $B=H_{1} \times H_{2}$ when we wish to distinguish the two direct factors. Note that $G^{+}=G \cap B$ and by the definition of $H$, the group $G^{+}$ projects onto $H_{1}$ and $H_{2}$. Let $g \in G \backslash G^{+}$. Then $g=(x, y)(12)$ for some $x, y \in H$, and since $G^{+}$projects onto $H_{2}$, multiplying $g$ by an element of $G^{+}$if necessary, we may assume that $y=1$ and $g=(x, 1)(12)$. Hence we may assume that $G=\left\langle G^{+}, g\right\rangle$ where $g=(x, 1)(12)$ for some $x \in H$. Since $G$ is elusive, there is no element of order 2 interchanging $\Delta_{1}$ and $\Delta_{2}$ (such an element would be fixed point free), and so $o(g) \neq 2$, in particular, $x \neq 1$.

We also need the following notation. Given a group $M$ and $\varphi \in \operatorname{Aut}(M)$, we denote the full diagonal subgroup $\left\{\left(a, a^{\varphi}\right) \mid a \in M\right\}$ of $M \times M$ by $\operatorname{Diag}_{\varphi}(M \times M)$. Also, given $G=\left\langle G^{+}, g\right\rangle$ as defined above, for $M \leqslant H_{1}$ we define $\operatorname{Diag}_{g}(M \times M)$ as the full diagonal subgroup $\left\{\left(a, a^{g}\right) \mid a \in M\right\}$. Moreover, we need the fact that $\operatorname{Aut}\left(T^{k}\right)=$ $\operatorname{Aut}(T)$ ? $S_{k}$ when $T$ is a nonabelian simple group.

\subsection{The examples}

We saw in Example 2.2 that the biquasiprimitive group $M_{10}=A_{6} \cdot 2$ acting on 12 points is elusive. We now give two further constructions of elusive biquasiprimitive Springer 
groups which we will see in Theorem 1.4 provide all the remaining biquasiprimitive elusive groups.

The following lemma concerns the second family of examples in Theorem 1.4.

Lemma 3.1. Let $G=M_{11}^{k} \rtimes K \leqslant M_{11} 2 S_{k}$, where $k$ is a positive integer and $K$ is a transitive subgroup of $S_{k}$ acting naturally on the $k$ simple direct factors of $M_{11}^{k}$. Suppose that $K$ contains an index 2 subgroup $K^{\prime}$ such that $K \backslash K^{\prime}$ contains no elements of order 2 and let $L=\operatorname{PSL}(2,11)^{k} \rtimes K^{\prime}$. Then the action of $G$ on the set of right cosets of $L$ in $G$ is faithful, biquasiprimitive and elusive of degree $2 \cdot(12)^{k}$.

Proof: Let $\Omega$ be the set of right cosets of $L$ in $G$ and let $N$ be the unique minimal normal subgroup of $G$. Then $N=M_{11}^{k}$ and $L N=M_{11}^{k} \rtimes K^{\prime}$. Let $G^{+}=L N$. Then $\left|G: G^{+}\right|=2$ and so $N$ has two orbits $\Delta_{1}$ and $\Delta_{2}$ on $\Omega$, each of size $12^{k}$. Hence every nontrivial normal subgroup of $G$ has at most two orbits on $\Omega$ and so $G$ is a biquasiprimitive permutation group of degree $2 \cdot(12)^{k}$.

By Corollary 2.4, each element of prime order in $G^{+}$fixes some point of $\Delta_{1}$. Since $K \backslash K^{\prime}$ contains no elements of order $2, G \backslash G^{+}$contains no elements of order 2 and hence contains no elements of prime order. Thus $G$ is elusive on $\Omega$.

An example of $\left(k, K, K^{\prime}\right)$ which satisfies the conditions of Lemma 3.1 is $k=4, K=$ $\langle(1234)\rangle$ and $K^{\prime}=\langle(13)(24)\rangle$. Another example is $k=5, K=Z_{5} \rtimes Z_{4}=\operatorname{AGL}(1,5)$ and $K^{\prime}=Z_{5} \rtimes Z_{2} \cong D_{10}$. Note that there is no requirement of transitivity for $K^{\prime}$.

Next we look at the 2-closure of $G$ when $G$ is of the above type.

Lemma 3.2. Suppose $G=M_{11}^{k} \rtimes K \leqslant M_{11} 2 S_{k}$ is a biquasiprimitive elusive group given by Lemma 3.1. Then the 2-closure of $G$ contains a fixed point free element of order 3 .

Proof: With the notation of Section 3.1, we may identify $\Omega=[G: L]$ with $\Delta \times\{1,2\}$ where $\Delta \times\{i\}=\Delta_{i}$. Let $N=M_{11}^{k}$. We observe that $N$ acts faithfully on each $\Delta_{i}$ inducing a product action on $\Delta$ with $\Delta=\Phi^{k}$, where $\Phi=\left[M_{11}: \operatorname{PSL}(2,11)\right]$ and $|\Phi|=$ 12. Let $M=N^{\Delta_{1}} \cong N$. Then $N=\operatorname{Diag}_{\varphi}(M \times M)$ where $\varphi=\left(\tau_{1}, \ldots, \tau_{k}\right) . \sigma \in$ $\operatorname{Aut}(M)$ for some $\tau_{i} \in M_{11}$ and $\sigma \in S_{k}$, and $N$ acts on $\Omega=\Delta \times\{1,2\}$ via $(\delta, 1)^{\left(g, g^{\varphi}\right)}=$ $\left(\delta^{g}, 1\right)$ and $(\delta, 2)^{\left(g, g^{\varphi}\right)}=\left(\delta^{g^{\varphi}}, 2\right)$. (For $g \in M=M_{11}^{k}$, we can write $g=\left(x_{1}, \ldots, x_{k}\right)$ where $x_{i} \in M_{11}$ for each $i$. Then $g^{\varphi}=\left(x_{1}^{\tau_{1}}, \ldots, x_{k}^{\tau_{k}}\right)^{\sigma}=\left(x_{1^{\prime}}^{\tau_{1^{\prime}}}, \ldots, x_{k^{\prime}}^{\tau_{k^{\prime}}}\right)$ where $i^{\prime}=$ $i^{\sigma^{-1}}$.)

The orbits of $N=\operatorname{Diag}_{\varphi}(M \times M)$ on the set $\Omega \times \Omega$ are of the following form.

1. $\{((\alpha, 1),(\beta, 1)):(\alpha, \beta) \in \mathcal{O}\}$, for each orbit $\mathcal{O}$ of $M$ on $\Delta \times \Delta$.

2. $\{((\alpha, 2),(\beta, 2)):(\alpha, \beta) \in \mathcal{O}\}$, for each orbit $\mathcal{O}$ of $M$ on $\Delta \times \Delta$.

3. $\left\{((\alpha, 1),(\beta, 2)):(\alpha, \beta) \in \mathcal{O}_{\gamma, \delta}^{\prime}\right\}$, where $\mathcal{O}_{\gamma, \delta}^{\prime}=\left\{\left(\gamma^{g}, \delta^{g^{\varphi}}\right): g \in M\right\}$ for some $\gamma, \delta \in \Delta$

4. $\left\{((\beta, 2),(\alpha, 1)):(\alpha, \beta) \in \mathcal{O}_{\gamma, \delta}^{\prime}\right\}$, where $\mathcal{O}_{\gamma, \delta}^{\prime}=\left\{\left(\gamma^{g}, \delta^{g^{\varphi}}\right): g \in M\right\}$ for some $\gamma, \delta \in \Delta$,

Since $\Delta=\Phi^{k}$, we write the points $\alpha=\left(\alpha_{1}, \ldots, \alpha_{k}\right)$ and $\beta=\left(\beta_{1}, \ldots, \beta_{k}\right)$ where $\alpha_{i}, \beta_{i} \in \Phi$. Let $y \in S_{12}$ be a fixed point free element of order 3 on 
$\Phi$. Recall that $\varphi=\left(\tau_{1}, \ldots, \tau_{k}\right) . \sigma$. Consider $h=\left((y, 1, \ldots, 1),\left(y^{\tau_{1}}, 1, \ldots, 1\right)^{\sigma}\right) \in$ $\operatorname{Diag}_{\varphi}\left(S_{12}^{k} \times S_{12}^{k}\right)$ acting on $\Delta \times\{1,2\}$. We will show that $h$ preserves $N$-orbits on ordered pairs, and hence $h$ is a fixed point free element of order 3 in $N^{(2)}$ on $\Omega$. By Lemma $2.8, G^{(2)} \geq N^{(2)}$, and hence $G^{(2)}$ also contains a fixed point free element of order 3 on $\Omega$.

By Proposition 2.9, the 2-closure of the product action $M=M_{11}^{k}$ on $\Delta=\Phi^{k}$ is $S_{12}^{k}$. Thus $h$ preserves $N$-orbits of types 1 and 2 . So we only need to check that $h$ preserves $N$-orbits of type 3 . (Note that the same argument will apply to the $N$-orbits of type 4.) Recall that $\varphi=\left(\tau_{1}, \ldots, \tau_{k}\right) . \sigma$ for $\sigma \in S_{k}$, and for convenience, write $\tau_{1}$ as $\tau$. Suppose $1^{\sigma}=i$, then

$$
h=\left((y, 1, \ldots, 1),\left(1, \ldots, 1, y^{\tau}, 1, \ldots, 1\right)\right) \text { where } y^{\tau} \text { is at the } i \text { th component. }
$$

Thus we only need to consider the action induced by the product action on the first component of $\Delta_{1}$ and the $i$ th component of $\Delta_{2}$. Then since we are only considering elements which act componentwise on $\Phi^{k}$ we may assume that $k=1$, that is, $\Delta=\Phi$. Let $\gamma, \delta \in \Delta$ and

$$
\Sigma=(\gamma, \delta)^{\left\{\left(g, g^{\tau}\right) \mid g \in M_{11}\right\}}=(\gamma, \delta)^{\operatorname{Diag}_{\tau}\left(M_{11} \times M_{11}\right)} .
$$

Then $\Sigma$ gives rise to an orbit of type 3 . Let $(\alpha, \beta) \in \Sigma$, that is,

$$
(\alpha, \beta)=(\gamma, \delta)^{\left(g_{1}, g_{1}^{\tau}\right)} \text { for some } g_{1} \in M_{11} \text {. }
$$

Applying $h=\left(y, y^{\tau}\right)$ to $(\alpha, \beta)$, we have

$$
(\alpha, \beta)^{\left(y, y^{\tau}\right)}=\left(\gamma^{g_{1} y}, \delta^{\tau^{-1} g_{1} y \tau}\right) .
$$

Then since $S_{12}=M_{11}^{(2)},(y, y)$ preserves the set

$$
\left(\gamma, \delta^{\tau^{-1}}\right)^{\left\{(g, g) \mid g \in M_{11}\right\}},
$$

and so there exists some $g_{2} \in M_{11}$, such that

$$
(\alpha, \beta)^{\left(y, y^{\tau}\right)}=\left(\gamma^{g_{2}}, \delta^{\tau^{-1} g_{2} \tau}\right) \in \Sigma
$$

Thus orbits of type 3 are fixed setwise by $h$, and hence $h$ is a fixed point free element of order 3 in $G^{(2)}$.

Next we look at the last family of examples described in Theorem 1.4.

Lemma 3.3. Let $G=M_{11}^{k} \rtimes K \leqslant M_{11} 2 S_{k}$, where $k$ is an even positive integer and $K$ is a transitive subgroup of $S_{k}$ acting naturally on the $k$ simple direct factors of $M_{11}^{k}$. Suppose that $K$ has an intransitive index 2 subgroup $K^{\prime}$ such that $K \backslash K^{\prime}$ contains no elements of order 2 and let $L=\left(\operatorname{PSL}(2,11)^{k / 2} \times M_{11}^{k / 2}\right) \rtimes K^{\prime}$. Then the action 祭 Springer 
of $G$ on the set of right cosets of $L$ in $G$ is faithful, biquasiprimitive and elusive of degree $2 \cdot(12)^{k / 2}$.

Proof: The results follow from the same argument as in the proof of Lemma 3.1.

An example of $\left(k, K, K^{\prime}\right)$ which satisfies the conditions of Lemma 3.3 is $k=4$, $K=\langle(1234)\rangle$ and $K^{\prime}=\langle(13)(24)\rangle$. The following lemma concerns the 2-closure of $G$ when $G$ is as in Lemma 3.3 .

Lemma 3.4. Suppose $G=M_{11}^{k} \rtimes K \leqslant M_{11} 2 S_{k}$ is a biquasiprimitive elusive group given by Lemma 3.3. Then $G^{(2)}$ contains a fixed point free element of order 3.

Proof: With the notation of Section 3.1, we may identify $\Omega=[G: L]$ with $\Delta \times\{1,2\}$ where $\Delta \times\{i\}=\Delta_{i}$. Let $N=M_{11}^{k}$ and write $N=M_{1} \times M_{2}$, where $M_{i} \cong M_{11}^{k / 2}$ for $i=1,2$, such that

$$
L=\left(\operatorname{PSL}(2,11)^{k / 2} \times M_{11}^{k / 2}\right) \rtimes K^{\prime}=\left(\operatorname{PSL}(2,11)^{k / 2} \times M_{2}\right) \rtimes K^{\prime}
$$

where PSL $(2,11)^{k / 2} \leqslant M_{1}$. Note that $M_{2} \triangleleft L$ and acts trivially on $\Delta_{1}:=[L N: L]$. Moreover, since elements of $G$ interchanging $\Delta_{1}$ and $\Delta_{2}$ also interchange $M_{1}$ and $M_{2}$, we have that $M_{1}$ acts trivially on $\Delta_{2}$. Thus $N=M_{1} \times M_{2}$ acts on $\Omega=\Delta \times\{1,2\}$ via the action $(\delta, i)^{\left(g_{1}, g_{2}\right)}=\left(\delta^{g_{i}}, i\right)$ for $i=1,2, \delta \in \Delta$ and $\left(g_{1}, g_{2}\right) \in M_{1} \times M_{2}$. Moreover each $M_{i}$ induces a faithful product action on $\Delta_{i}=\Delta \times\{i\}$, that is, $\Delta=\Phi^{k / 2}$ where $\Phi=\left[M_{11}: \operatorname{PSL}(2,11)\right]$ and $\left|\Delta_{i}\right|=12^{k / 2}$. Then for each $i, M_{i} \cong N^{\Delta_{i}}$ and so $M:=$ $N^{\Delta_{1}} \cong M_{11}^{k / 2}$.

The orbits of $N$ on the set $\Omega \times \Omega$ are of the following form.

1. $\{((\alpha, 1),(\beta, 1)):(\alpha, \beta) \in \mathcal{O}\}$ for each orbit $\mathcal{O}$ of $M$ on $\Delta \times \Delta$.

2. $\{((\alpha, 2),(\beta, 2)):(\alpha, \beta) \in \mathcal{O}\}$ for each orbit $\mathcal{O}$ of $M$ on $\Delta \times \Delta$.

3. $\{((\alpha, 1),(\beta, 2)):(\alpha, \beta) \in \Delta \times \Delta\}$.

4. $\{((\beta, 2),(\alpha, 1)):(\beta, \alpha) \in \Delta \times \Delta\}$.

By Proposition 2.9, the 2-closure of the product action of $M=M_{11}^{k / 2}$ on $\Delta=\Phi^{k / 2}$ is $S_{12}^{k / 2}$. Let $h=\left(g_{1}, g_{2}\right) \in S_{12}^{k / 2} \times S_{12}^{k / 2}$. Then $h$ acts on $\Omega$ by $(\delta, i)^{h}=\left(\delta^{g_{i}}, i\right)$. Moreover, $h$ fixes each orbit of $N$ on $\Omega \times \Omega$ and so $S_{12}^{k} \leqslant N^{(2)}$. By Lemma $2.9, N^{(2)} \leqslant G^{(2)}$. Thus $G^{(2)}$ contains many fixed point free elements of prime order, in particular, a fixed point free element of order 3 .

\section{Determining all biquasiprimitive elusive groups}

Throughout this section, we use the notation of Section 3.1. Let $G$ be a biquasiprimitive elusive group, and let $\Delta_{1}, \Delta_{2}$ be the two orbits of $G^{+}$.

Lemma 4.1. Let $G$ be an elusive biquasiprimitive permutation group and let $N$ be a minimal normal subgroup of $G$. Then $N \leqslant G^{+}$. 
Proof: Since $N$ is a minimal normal subgroup of $G$, either $N \leqslant G^{+}$or $N \cap G^{+}=1$. If $N \cap G^{+}=1$, then $G=G^{+} \times N$ where $N=\langle g\rangle$ and $g$ is an element of order 2 interchanging $\Delta_{1}$ and $\Delta_{2}$, which is not the case as $G$ is elusive. Thus $N \leqslant G^{+}$.

\subsection{The case where $G^{+}$acts faithfully on both orbits}

In this subsection, we consider the case where $G^{+}$acts faithfully on each orbit $\Delta_{i}$ for $i=1,2$.

Lemma 4.2. Let $G$ be an elusive biquasiprimitive group and suppose $G^{+}$acts faithfully on both orbits $\Delta_{i}$. Then $G$ has a unique minimal normal subgroup $N$ isomorphic to $T^{k}$ for some nonabelian simple group $T$ and a positive integer $k$.

Proof: Let $N$ and $M$ be distinct minimal normal subgroups of $G$. By Lemma 4.1, we have $N, M \leqslant G^{+}$and so $N^{\Delta_{1}}, M^{\Delta_{1}} \triangleleft\left(G^{+}\right)^{\Delta_{1}}$. Since $N \cap M=1$ and $G^{+}$is faithful on $\Delta_{1}$, it follows that $N^{\Delta_{1}} \cong N$ and $M^{\Delta_{1}} \cong M$, and we have $N^{\Delta_{1}} \cap M^{\Delta_{1}}=1$. Also biquasiprimitivity of $G$ implies that $N^{\Delta_{1}}$ and $M^{\Delta_{1}}$ are transitive. Thus by Lemma 2.11, $N^{\Delta_{1}}$ and $M^{\Delta_{1}}$ are regular. The same argument shows that $N^{\Delta_{2}}$ and $M^{\Delta_{2}}$ are regular. Then as both $N$ and $M$ are faithful on $\Delta_{1}$ and $\Delta_{2}$ it follows that both $N$ and $M$ are semiregular, contradicting $G$ being elusive. Thus $G$ has a unique minimal normal subgroup $N$. Suppose now that $N$ is abelian. Then $N^{\Delta_{1}}$ and $N^{\Delta_{2}}$ are regular and since $G^{+}$is faithful on both orbits, $N$ is semiregular on $\Omega$. This contradicts $G$ being elusive and so $N \cong T^{k}$ for some nonabelian simple group $T$.

Let $N \cong T^{k}$ be the minimal normal subgroup of $G$. We denote the $j$ th simple direct factor of $N$ by $T_{j}$ and write $N=T_{1} \times \cdots \times T_{k}$ where each $T_{j} \cong T$. Let $\alpha \in \Delta_{1}$ and let $N_{\alpha}$ be the stabiliser in $N$ of $\alpha$. We will first determine $N$ and $N_{\alpha}$. For each $j \in\{1, \ldots, k\}$, let $\Pi_{j}: N \rightarrow T_{j}$ denote the projection onto the $j$ th simple direct factor of $N$, and let

$$
N_{j}:=N_{\alpha} \cap T_{j}
$$

Then $N_{j} \unlhd N_{\alpha}$, and hence

$$
N_{j} \cong \Pi_{j}\left(N_{j}\right) \unlhd \Pi_{j}\left(N_{\alpha}\right), \quad \text { for each } j
$$

The next lemma is true for all elusive groups.

Lemma 4.3. Let $G$ be an elusive group on a set $\Omega$ and let $N$ be a normal subgroup of $G$ such that $N \cong T^{k}$ for some non-abelian simple group $T$. Let $\alpha \in \Omega$. Then for each $j \in\{1, \ldots, k\}$, the projection $\Pi_{j}\left(N_{\alpha}\right)$ either equals $T_{j}$ or is a proper subgroup of $T_{j}$ which meets every $\operatorname{Aut}\left(T_{j}\right)$-conjugacy class of elements of $T_{j}$ of prime order. 
Proof: Suppose that there exists $j \in\{1, \ldots, k\}$ such that $\Pi_{j}\left(N_{\alpha}\right) \neq T_{j}$. For each $\operatorname{Aut}(T)$-conjugacy class $C$ of elements of prime order in $T$, the set

$$
\bar{C}=\left\{\left(t_{1}, \ldots, t_{k}\right): t_{i} \in C\right\}
$$

is an $\operatorname{Aut}(N)$-conjugacy class of elements of prime order in $N$. Then as $G$ is elusive, $\bar{C} \cap N_{\alpha} \neq \varnothing$. Hence the projection $\Pi_{j}\left(N_{\alpha}\right)$ is a proper subgroup of $T_{j}$ which meets every $\operatorname{Aut}\left(T_{j}\right)$-conjugacy class of elements of $T_{j}$ of prime order.

Lemma 4.4. Let $G$ be a biquasiprimitive elusive group such that $G^{+}$is faithful on both orbits, and let $N$ be the unique minimal normal subgroup of $G$. With the notation in the paragraph preceding Lemma 4.3 , there exists at least one $i \in\{1, \ldots, k\}$ such that $\Pi_{i}\left(N_{\alpha}\right)$ is a proper subgroup of $T_{i}$ which meets every $\operatorname{Aut}\left(T_{i}\right)$-conjugacy class of $T_{i}$. In addition,

1. $T=A_{6}, M_{11}, \mathrm{P} \Omega^{+}(8,2)$ or $\mathrm{P} \Omega^{+}(8,3)$, and $\Pi_{i}\left(N_{\alpha}\right) \cong A_{5}, \operatorname{PSL}(2,11), \operatorname{P} \Omega(7,2)$ or $\mathrm{P} \Omega(7,3)$, respectively. In particular, $\Pi_{i}\left(N_{\alpha}\right)$ is a nonabelian simple group.

2. $N_{i} \cong \Pi_{i}\left(N_{\alpha}\right)$.

\section{Proof:}

(1) Since $G^{+}$is faithful on $\Delta_{1}, N \leqslant G^{+}$(by Lemma 4.1) is faithful on $\Delta_{1}$, and hence $N_{\alpha}$ does not contain any of the simple direct factors of $N$. Now let $t \in T$ have prime order, then $(t, 1, \ldots, 1) \in N$ has prime order. Let $C$ be the $G$-conjugacy class containing the element $(t, 1, \ldots, 1)$. Since $G$ is elusive and $C \subset N$, we have $C \cap N_{\alpha} \neq \varnothing$. Thus, there exists $i \in\{1, \ldots, k\}$ such that $N_{i} \neq 1$, and hence $\Pi_{i}\left(N_{i}\right) \neq 1$. Since $T$ is simple and $N_{\alpha}$ does not contain any of the simple direct factors of $N$, together with $(*)$, we have $\Pi_{i}\left(N_{\alpha}\right)$ is a proper subgroup of $T$. By Lemma $4.3, \Pi_{i}\left(N_{\alpha}\right)$ meets every $\operatorname{Aut}\left(T_{i}\right)$-conjugacy class of elements of prime order in $T_{i}$. By Theorem 2.7, we have $T=A_{6}, M_{11}, \mathrm{P}^{+}(8,2)$ or $\mathrm{P} \Omega^{+}(8,3)$, and $\Pi_{i}\left(N_{\alpha}\right) \cong A_{5}, \operatorname{PSL}(2,11), \mathrm{P} \Omega(7,2)$ or $\mathrm{P} \Omega(7,3)$, respectively.

(2) Note that $\Pi_{i}\left(N_{\alpha}\right)$ is simple. Hence $(*)$ and the fact that $\Pi_{i}\left(N_{i}\right) \neq 1$ combined together imply that $N_{i} \cong \Pi_{i}\left(N_{\alpha}\right)$.

Lemma 4.5. Suppose $G$ is an elusive biquasiprimitive group on a set $\Omega$ and $G^{+}$acts faithfully on its two orbits. Let $N$ be the unique minimal normal subgroup of $G$ and suppose that $N \cong T^{k}$ for some nonabelian simple group $T$. Then for each $\alpha \in \Omega$, we have $N_{\alpha} \cong R^{k}$, where $(T, R)$ is one of $\left(A_{6}, A_{5}\right),\left(M_{11}, \operatorname{PSL}(2,11)\right),\left(\operatorname{P} \Omega^{+}(8,2), \operatorname{P} \Omega(7,2)\right)$ or $\left(\mathrm{P} \Omega^{+}(8,3), \mathrm{P} \Omega(7,3)\right)$.

Proof: By Lemma 4.4, we have that $N=T_{1} \times \cdots \times T_{k}$ where each $T_{i} \cong T \in$ $\left\{A_{6}, M_{11}, \mathrm{P} \Omega^{+}(8,2), \mathrm{P} \Omega^{+}(8,3)\right\}$. Without loss of generality, we may suppose $\alpha \in \Delta_{1}$. Since $N$ is transitive on $\Delta_{1}$, we have $G^{+}=N G_{\alpha}$. Also since $N$ is a minimal normal subgroup of $G, G$ acts transitively by conjugation on the set of $k$ simple direct factors of $N$. Note that $G^{+}$is an index 2 subgroup of $G$, hence $G^{+}$either acts transitively or has two equal length orbits on the set of $k$ simple direct factors of $N$.

First we suppose that $G^{+}$is transitive on the set of $k$ simple direct factors of $N$. Since $G^{+}=N G_{\alpha}, G_{\alpha}$ is also transitive on the set of $k$ simple direct factors of $N$. So for 
each $i, j \in\{1, \ldots, k\}$, we have $\Pi_{i}\left(N_{\alpha}\right) \cong \Pi_{j}\left(N_{\alpha}\right)$. By Lemma $4.4, \Pi_{j}\left(N_{\alpha}\right) \neq T_{j}$ for each $j$. Moreover, by Lemma 4.4 (2), we have $N_{j}=N_{\alpha} \cap T_{j} \cong \Pi_{j}\left(N_{\alpha}\right)$ for each $j$. Hence $N_{\alpha}=N_{1} \times \cdots \times N_{k} \cong R^{k}$ where $(T, R)$ is one of $\left(A_{6}, A_{5}\right),\left(M_{11}, \operatorname{PSL}(2,11)\right)$, $\left(\mathrm{P} \Omega^{+}(8,2), \mathrm{P} \Omega(7,2)\right)$ or $\left(\mathrm{P} \Omega^{+}(8,3), \mathrm{P} \Omega(7,3)\right)$. The result holds in this case.

Secondly we suppose that $G^{+}$, and hence $G_{\alpha}$, have two orbits, say, $\mathcal{O}_{1}=$ $\left\{T_{1}, \ldots, T_{k / 2}\right\}$ and $\mathcal{O}_{2}=\left\{T_{(k / 2)+1}, \ldots, T_{k}\right\}$ on the set of $k$ simple direct factors of $N$. Thus, $\Pi_{i}\left(N_{\alpha}\right) \cong \Pi_{j}\left(N_{\alpha}\right)$ if $i, j \in\{1, \ldots, k / 2\}$ or $i, j \in\{(k / 2)+1, \ldots, k\}$. By Lemma 4.4, there exists $i$ such that $\Pi_{i}\left(N_{\alpha}\right) \neq T_{i}$ and $N_{i}=N_{\alpha} \cap T_{i} \cong \Pi_{i}\left(N_{\alpha}\right)$. Moreover $\Pi_{i}\left(N_{\alpha}\right)$ is determined by $T$. Without loss of generality, we may assume $T_{i} \in \mathcal{O}_{1}$. Then as $G_{\alpha}$ is transitive on $\mathcal{O}_{1}$, it follows that $N_{1} \times \cdots \times N_{k / 2} \leqslant N_{\alpha}$ and $N_{1} \times \cdots \times N_{k / 2} \cong R^{k / 2}$ where $(T, R)$ is one of $\left(A_{6}, A_{5}\right),\left(M_{11}, \operatorname{PSL}(2,11)\right)$, $\left(\mathrm{P} \Omega^{+}(8,2), \mathrm{P} \Omega(7,2)\right)$ or $\left(\mathrm{P} \Omega^{+}(8,3), \mathrm{P} \Omega(7,3)\right)$. Moreover, by Lemma 4.3 , for each $j \in\{(k / 2)+1, \ldots, k\}$, either $\Pi_{j}\left(N_{\alpha}\right) \cong R$ or $\Pi_{j}\left(N_{\alpha}\right)=T_{j}$. Let $t \in T$ have prime order, and let $C$ be the $G$-conjugacy class of the prime order element $(t, 1, \ldots, 1, t)$. Note that $C \cap N_{\alpha} \neq \varnothing$. Since $G$ preserves the partition $\left\{\mathcal{O}_{1}, \mathcal{O}_{2}\right\}$ of $\left\{T_{1}, \ldots, T_{k}\right\}$, every element of $C$ has precisely one nontrivial coordinate in $\mathcal{O}_{1}$ and precisely one nontrivial coordinate in $\mathcal{O}_{2}$. Thus, as $N_{1} \times \cdots \times N_{k / 2} \leqslant N_{\alpha}$ and for each $i \in\{1, \ldots, k / 2\}$ we have $\Pi_{i}\left(N_{\alpha}\right) \cong N_{i}$, it follows that there exists $j \in\{(k / 2)+1, \ldots, k\}$ such that $N_{j} \neq 1$. By $(*), 1 \neq \Pi_{j}\left(N_{j}\right) \unlhd \Pi_{j}\left(N_{\alpha}\right)$. However, $T$ and $R$ are simple. Hence $N_{j} \cong T$ or $R$. But $N_{\alpha}$ does not contain any of the simple direct factors of $N$ (as $N$ is faithful on $\left.\Delta_{1}\right)$, so $\Pi_{j}\left(N_{\alpha}\right) \cong R$ and $N_{j} \cong R$. Since $G_{\alpha}$ is transitive on $\mathcal{O}_{2}$, it follows that $N_{\alpha} \cong R^{k}$. Thus the assertion holds in this case too.

Finally, we determine all biquasiprimitive elusive groups $G$ where $G^{+}$acts faithfully on both orbits.

Proposition 4.6. Suppose that $G$ is an elusive biquasiprimitive group on a set $\Omega$, and $G^{+}$acts faithfully on both orbits. Let $N$ be a minimal normal subgroup of $G$. Then $N \cong T^{k}$ where $T=M_{11}$ or $A_{6}$. Moreover, the following results hold.

(a) If $T=A_{6}$, then $G=M_{10}$ acting on 12 points.

(b) If $T=M_{11}$, then $G$ is given by Lemma 3.1, that is, $G$ satisfies:

(1) $G=M_{11}^{k} \rtimes K \leqslant M_{11} 2 S_{k}$ where $K \leqslant S_{k}$ acts transitively by permuting the $k$ simple direct factors of $M_{11}^{k}$.

(2) $G^{+}=M_{11}^{k} \rtimes K^{\prime}$ where $\left|K: K^{\prime}\right|=2$ and $K \backslash K^{\prime}$ contains no elements of order 2 .

(3) For $\alpha \in \Omega, G_{\alpha} \cong \operatorname{PSL}(2,11)^{k} \rtimes K^{\prime}$, and $|\Omega|=2 \cdot 12^{k}$.

Proof: By Lemma 4.4, $N \cong T^{k}$ where $T=A_{6}, M_{11}, \mathrm{P} \Omega^{+}(8,2)$ or $\mathrm{P} \Omega^{+}(8,3)$. Moreover, by Lemma 4.2, $N$ is the unique minimal normal subgroup of $G$ and so $C_{G}(N)=1$. Thus $G \leqslant \operatorname{Aut}(N)=\operatorname{Aut}(T)$ ? $S_{k}$. Let $\alpha \in \Omega$. Then by Lemma $4.5, N_{\alpha} \cong R^{k}$ where $R=A_{5}, \operatorname{PSL}(2,11), \operatorname{P} \Omega(7,2)$ or $\mathrm{P} \Omega(7,3)$, respectively.

With the notation of Section 3.1, since $G^{+}$acts faithfully on both orbits $\Delta_{1}$ and $\Delta_{2}$, $G^{+}=\operatorname{Diag}_{\varphi}(H \times H)$ where $H=\left(G^{+}\right)^{\Delta_{1}}$ and $\varphi \in \operatorname{Aut}(H)$. Identifying $\Omega=\Delta \times$ $\{1,2\}$, the action of $G$ is given by (3.1) (see Section 3.1). Let $M=N^{\Delta_{1}} \triangleleft H$. Then $M \cong T^{k}$. Since $C_{G}(N)=1, \varphi$ induces a nontrivial automorphism of $M$, and so we also write $N=\operatorname{Diag}_{\varphi}(M \times M)$ where $\varphi \in \operatorname{Aut}(M)$. Note that, when $T=A_{6}$, there Springer 
are two conjugacy classes of subgroups isomorphic to $A_{5}$; when $T=M_{11}$, there is one conjugacy class of subgroups isomorphic to $\operatorname{PSL}(2,11)$; when $T=\mathrm{P} \Omega^{+}(8,2)$, there are three conjugacy classes of subgroups isomorphic to $\operatorname{P} \Omega(7,2)$; while when $T=\mathrm{P} \Omega^{+}(8,3)$, there are six conjugacy classes of subgroups isomorphic to $\mathrm{P} \Omega(7,3)$. However, in all four cases there is a unique class of subgroups isomorphic to $R$ under $\operatorname{Aut}(T)$. Hence the stabiliser of a point in $N^{\Delta_{1}}$ is conjugate under $\operatorname{Aut}(N)$ to $R^{k}$. Thus we may assume $N^{\Delta_{1}}=M=T^{k}$, with point stabiliser $M_{\alpha}=R^{k}$, acting on $\Delta_{1}=\Phi^{k}$ with $|\Phi|=|T: R|$ as a natural product action.

Suppose first that $T=\mathrm{P} \Omega^{+}(8,2)$ or $\mathrm{P} \Omega^{+}(8,3)$, then by Lemma $4.4, R=\mathrm{P} \Omega(7,2)$ or $\operatorname{P} \Omega(7,3)$. There are three conjugacy classes of elements of order 5 in $T$. Using the permutation characters and character tables in [4, pp. 85-87, pp. 136-140], we see that precisely two of the conjugacy classes do not meet $R$. Thus, given $\varphi \in \operatorname{Aut}\left(T^{k}\right)$, say, $\varphi=\left(\tau_{1}, \ldots, \tau_{k}\right) . \sigma$, for $\tau_{i} \in \operatorname{Aut}(T)$ and $\sigma \in S_{k}$, we can find two elements $y_{1}, y_{2}$ of order 5 , such that $y_{1}^{\tau_{1}}=y_{2}$, and the conjugacy classes of $y_{1}$ and $y_{2}$ do not meet $R$. Thus

$$
h=\left(\left(y_{1}, 1, \ldots, 1\right),\left(y_{1}, 1, \ldots, 1\right)^{\varphi}\right)
$$

is a fixed point free element of order 5 in $N$, which is a contradiction. Thus $T$ cannot be $\mathrm{P} \Omega^{+}(8,2)$ or $\mathrm{P} \Omega^{+}(8,3)$. Therefore, $T=A_{6}$ or $T=M_{11}$ as asserted.

(a) We first consider the case that $T=A_{6}$. Recall that $N^{\Delta_{1}}=M=T^{k}$ acts on $\Delta_{1}=\Phi^{k}$ with $|\Phi|=|T: R|$ as a natural product action with point stabiliser $M_{\alpha}=R^{k}$. Then in this case we have $|\Phi|=6$. There are two conjugacy classes of elements of order 3 in $A_{6}$, one with cycle structure $3^{2}$, another with cycle structure $3.1^{3}$. They are interchanged by an outer automorphism of $S_{6}$. For each $j \in\{1, \ldots, k\}$, consider an element $h_{j} \in N$ of order 3 where $h_{j}^{\Delta_{1}}=\left(1, \ldots, 1, y_{j}, 1, \ldots, 1\right)$ such that $y_{j} \in T_{j}$ has cycle structure $3^{2}$ and $T_{j}$ is the $j$ th direct factor of $M$. Then

$$
h_{j}=\left(\left(1, \ldots, 1, y_{j}, 1, \ldots, 1\right),\left(1, \ldots, 1, y_{j}, 1, \ldots, 1\right)^{\varphi}\right) .
$$

Since $h_{j}^{\Delta_{1}}$ is fixed point free and $G$ is elusive, we have $\left(1, \ldots, 1, y_{j}, 1, \ldots, 1\right)^{\varphi}$ must have a fixed point on $\Delta_{2}$. This means $\varphi=\left(\tau_{1}, \ldots, \tau_{k}\right) . \sigma$ where each $\tau_{i} \in \operatorname{Aut}\left(A_{6}\right)$ interchanges the two conjugacy classes of elements of order 3 in $T$, and $\sigma \in S_{k}$. Now if $k \geq 2$, consider the following element of order 3 ,

$$
h=\left((y, z, 1, \ldots, 1),(y, z, 1, \ldots, 1)^{\varphi}\right),
$$

where $y \in A_{6}$ with cycle structure $3^{2}$ and $z \in A_{6}$ with cycle structure $3.1^{3}$. It is easy to see that $h$ is fixed point free on $\Omega$. Hence $k=1$, and $G$ is almost simple. By [13, Page 83], $G=M_{10}$ acting on 12 points. The proof of (a) is complete.

(b) This leaves us with the final case where $T=M_{11}$ and the unique minimal normal subgroup $N \cong T^{k}$ for $k \geq 1$.

Since $N$ is the unique minimal normal subgroup of $G, G \leqslant \operatorname{Aut}(N)=M_{11}^{k} \rtimes S_{k}$ where the top group $S_{k}$ acts by permuting the $k$ simple direct factors of $N$. That is, there exists a subgroup $K \leqslant S_{k}$ such that $G=M_{11}^{k} \rtimes K$. Since $N$ is a minimal normal subgroup, $K$ is transitive on the set of $k$ simple direct factors. Thus condition (1) holds.

By Lemma 4.1, $N \leqslant G^{+}$, and so $G^{+}=M_{11}^{k} \rtimes K^{\prime}$ where $\left|K: K^{\prime}\right|=2$. Since $G$ is elusive, $G \backslash G^{+}$contains no elements of order 2 . Hence $K \backslash K^{\prime}$ contains no elements of order 2. Thus condition (2) holds. 
The biquasiprimitivity implies that $N$ and $G^{+}$have the same two orbits. By Lemma 4.5, we have $N_{\alpha} \cong \operatorname{PSL}(2,11)^{k}$. Thus $N$ induces a product action on each orbit and $|\Omega|=2 \cdot 12^{k}$. Now conditions (1) and (2) imply that $G_{\alpha}=\operatorname{PSL}(2,11)^{k} \rtimes K^{\prime} \leqslant$ $G^{+}$, so condition (3) holds and the proof of (b) is complete too.

\subsection{The case where $G^{+}$is not faithful on its orbits}

Lemma 4.7. Let $G$ be a biquasiprimitive elusive group acting on a set $\Omega$ such that $G^{+}$does not act faithfully on at least one of its orbits. Then $G$ has a unique minimal normal subgroup $N$. Moreover $N \cong M_{11}^{k}$ with $k$ even and for $\alpha \in \Omega$, we have $N_{\alpha} \cong$ $\operatorname{PSL}(2,11)^{k / 2} \times M_{11}^{k / 2}$ and $|\Omega|=2 \cdot 12^{k / 2}$.

Proof: Denote the two orbits of $G^{+}$on $\Omega$ by $\Delta_{1}$ and $\Delta_{2}$. For $i=1,2$, let $K_{i}$ be the kernel of the action of $G^{+}$on $\Delta_{i}$. Then $K_{1}$ acts faithfully on $\Delta_{2}$ and $K_{2}$ acts faithfully on $\Delta_{1}$, and at least one of $K_{1}, K_{2}$ is nontrivial. Moreover, each $g \in G \backslash G^{+}$ interchanges $K_{1}$ and $K_{2}$, and so $K_{1} \times K_{2} \triangleleft G$. In particular, $K_{1} \cong K_{2} \neq 1$. By the biquasiprimitivity of $G$ it follows that $K_{1}$ is transitive on $\Delta_{2}$ and $K_{2}$ is transitive on $\Delta_{1}$. Now $K_{1} \triangleleft G^{+}$and so contains a minimal normal subgroup $M_{1}$ of $G^{+}$. Then for any $g \in G \backslash G^{+}, M_{1}^{g} \leqslant K_{2}$ and $M_{1}^{g} \triangleleft G^{+}$. Moreover $g^{2} \in G^{+}$and hence normalises $M_{1}$, so $\left(M_{1}^{g}\right)^{g}=M_{1}$. Hence $N:=M_{1} \times M_{1}^{g}$ is a minimal normal subgroup of $G$ and by the biquasiprimitivity of $G$ it follows that $M_{1}$ is transitive on $\Delta_{2}$ and $M_{1}^{g}$ is transitive on $\Delta_{1}$. Suppose $M_{1}$ contains an element $h$ of prime order which is fixed point free on $\Delta_{2}$. Then $h^{g}$ is fixed point free on $\Delta_{1}$, and hence $\left(h, h^{g}\right) \in N$ is fixed point free on $\Omega$. As $G$ is elusive, it follows that $M_{1}$ is elusive on $\Delta_{2}$. Hence by Proposition 2.6, $M_{1} \cong M_{11}^{k / 2}$ for some even integer $k$, and for $\alpha \in \Delta_{2},\left(M_{1}\right)_{\alpha} \cong \operatorname{PSL}(2,11)^{k / 2}$. Hence $\left|\Delta_{2}\right|=12^{k / 2}$. It follows that $N \cong M_{11}^{k},|\Omega|=2 \cdot 12^{k / 2}$ and for all $\alpha \in \Omega, N_{\alpha} \cong \operatorname{PSL}(2,11)^{k / 2} \times M_{11}^{k / 2}$.

Moreover, for $\alpha \in \Delta_{2}$, we have that $\left(M_{1}\right)_{\alpha}$ is self-normalising in $M_{1}$. Thus by Lemma $2.11, C_{\operatorname{Sym}\left(\Delta_{2}\right)}\left(M_{1}\right)=1$ and so $\operatorname{Soc}\left(\left(G^{+}\right)^{\Delta_{2}}\right)=M_{1}$. Let $H=\left(G^{+}\right)^{\Delta_{2}}$. Then by [30, Lemma 3.2], $\operatorname{Soc}(G) \leqslant \operatorname{Soc}(H) \times \operatorname{Soc}(H)$. Hence $G$ has a unique minimal normal subgroup $N=M_{1} \times M_{1}^{g}$ as asserted.

Finally we determine all biquasiprimitive elusive groups $G$ where $G^{+}$does not act faithfully on at least one of its orbits.

Lemma 4.8. Suppose $G$ is a biquasiprimitive elusive group on $\Omega$ and $G^{+}$does not act faithfully on at least one of its orbits. Then $G$ is as given by Lemma 3.3, that is, $G$ satisfies the following conditions.

(1) $G=M_{11}^{k} \rtimes K \leqslant M_{11} 2 S_{k}$ where $K \leqslant S_{k}$ is transitive.

(2) $G^{+}=M_{11}^{k} \rtimes K^{\prime}$ where $\left|K: K^{\prime}\right|=2$, and $K^{\prime}$ is intransitive. Moreover there are no elements of order 2 in $K \backslash K^{\prime}$.

(3) For any $\alpha \in \Omega$, we have $G_{\alpha} \cong\left(\operatorname{PSL}(2,11)^{k / 2} \times M_{11}^{k / 2}\right) \rtimes K^{\prime}$ and $|\Omega|=2 \cdot 12^{k / 2}$.

Proof: By Lemma 4.7, $\operatorname{Soc}(G)=M_{11}^{k}$ is the unique minimal normal subgroup of $G$, and hence we have condition (1) satisfied. Now $G^{+}$is an index 2 subgroup of $G$, so we have $G^{+}=M_{11}^{k} \rtimes K^{\prime}$ and $\left|K: K^{\prime}\right|=2$. Moreover, by the structure of $G_{\alpha}$, the group $K^{\prime}$ is intransitive. Also, since $G$ is elusive, $G \backslash G^{+}$contains no elements of order 2, Springer 
and so $K \backslash K^{\prime}$ contains no elements of order 2 . Finally, by Lemma 4.7, $|\Omega|=2 \cdot 12^{k / 2}$ and hence $G_{\alpha} \cong\left(\operatorname{PSL}(2,11)^{k / 2} \times M_{11}^{k / 2}\right) \rtimes K^{\prime}$.

Now we are ready to prove Theorem 1.4 .

Proof of Theorem 1.4: First note that by Example 2.2, Lemmas 3.1 and 3.3, each group $G$ in (1)-(3) is biquasiprimitive and elusive. Now let $G$ be a biquasiprimitive elusive permutation group on $\Omega$. Suppose first that $G^{+}$is faithful on its orbits. Then by Proposition 4.6, either $G=M_{10}$ acting on 12 points or $G$ is of the type given in Lemma 3.1. In the first case, Example 2.2 shows that $M_{10}^{(2)}$ contains $A_{6} \times A_{6}$, and hence contains a fixed point free element of order 3. In the latter case, by Lemma 3.2, $G^{(2)}$ contains a fixed point free element of order 3. Next we suppose that $G^{+}$is not faithful on at least one of its orbits. By Lemma 4.8, $G$ is as in Lemma 3.3. Then by Lemma 3.4, $G^{(2)}$ contains a fixed point free element of order 3 . The proof is complete.

\section{Proofs of Theorems 1.1 and 1.3}

Proof of Theorem 1.1: We may assume that $\Gamma$ is connected. First suppose that there exists a non-trivial normal subgroup $N$ of $G$ such that $N$ has at least 3 orbits on the vertex set $V \Gamma$ of the graph $\Gamma$. By Theorem 2.10, $N$ is semiregular on $V \Gamma$ and so contains fixed point free elements of prime order. Next suppose that every non-trivial normal subgroup is transitive on $V \Gamma$. Then $G$ is quasiprimitive on $V \Gamma$. So by Theorem 2.5 , either $G$ contains a fixed point free element of prime order or $|V \Gamma|=12^{k}$ and $G=$ $M_{11} 2 K$ for some transitive subgroup $K \leqslant S_{k}$. In the latter case, by Proposition 2.9, the 2-closure of $M_{11}^{k}$ on $\Omega$ is $S_{12}^{k}$. By Lemma $2.8, S_{12} 2 K \leqslant G^{(2)} \leqslant \operatorname{Aut}(\Gamma)^{(2)}=\operatorname{Aut}(\Gamma)$. Thus $\Gamma$ has a fixed point free automorphism of order 3 .

We are left to deal with the case where every nontrivial normal subgroup of $G$ has at most 2 orbits on $V \Gamma$ and there exists one nontrivial normal subgroup with precisely 2 orbits, that is, $G$ is biquasiprimitive on $V \Gamma$. Either $G$ contains a fixed point free element of prime order, or $G$ is an elusive biquasiprimitive group. In the latter case, by Theorem 1.4, $G^{(2)}$ is not elusive. Hence by Lemma $2.8, G^{(2)} \leqslant \operatorname{Aut}(\Gamma)^{(2)}=\operatorname{Aut}(\Gamma)$, and $\Gamma$ has a fixed point free automorphism of prime order.

Proof of Theorem 1.3: Consider the orbital (undirected) graph $\Gamma$ of $G$ relative to $\Sigma$ (note that $\Sigma$ is self paired) with vertex set $\Omega$ and edge set $(\omega, \alpha)^{G}$ for $\alpha \in \Sigma$. By assumption, $G \leqslant \operatorname{Aut}(\Gamma)$ is vertex-transitive and locally-quasiprimitive. Now by the proof of Theorem 1.1, we have $G^{(2)} \leqslant \operatorname{Aut}(\Gamma)$ contains a fixed point free element of prime order. The proof is complete.

Acknowledgments The authors are grateful to Prof. Cheryl E. Praeger and Dr. Cai Heng Li for many helpful discussions, and to the referees for suggestions which greatly improved the paper.

\section{References}

1. N. Biggs, "Three remarkable graphs," Canad. J. Math. 25 (1973), 397-411.

2. P.J. Cameron, M. Giudici, G.A. Jones, W.M. Kantor, M.H. Klin, D. Marušič, and L.A. Nowitz, "Transitive permutation groups without semiregular subgroups," J. London Math. Soc. 66 (2002), 325-333.

3. P.J. Cameron (ed.), "Problems from the fifteenth British combinatorial conference," Discrete Math. 167/168 (1997), 605-661. 
4. J.H. Conway, R.T. Curtis, S.P. Norton, R.A. Parker and R.A. Wilson, Atlas of Finite Groups, Clarendon Press, Oxford, 1985.

5. J.D. Dixon and B. Mortimer, Permutation Groups, Springer-Verlag, New York-Heidelberg-Berlin, 1996.

6. E. Dobson, A. Malnič, D. Marušič, and L.A. Nowitz, "Minimal normal subgroups of transitive permutation groups of square-free degree", to appear in Discrete Math.

7. E. Dobson, A. Malnič, D. Marušič, and L.A. Nowitz, "Semiregular automorphisms of vertex-transitive graphs of certain valencies", to appear in J. Combin. Theory Ser. B.

8. S.F. Du, J.H. Kwak, and M.Y. Xu, "2-arc-transitive regular covers of complete graphs having the covering transformation group $Z_{p}^{3}$," J. Combin. Theory Ser. B. 93 (2005), 73-93.

9. S.F. Du, D. Marušič, and A.O. Waller, "On 2-arc-transitive covers of complete graphs," J. Combin. Theory Ser. B. 74 (1998), 276-290.

10. X.G. Fang and C.E. Praeger, "Finite two-arc transitive graphs admitting a Suzuki simple group," Comm. Algebra 27 (1999), 3727-3754.

11. X.G. Fang and C.E. Praeger, "Finite two-arc transitive graphs admitting a Ree simple group," Comm. Algebra 27 (1999), 3755-3769.

12. B. Fein, W.M. Kantor, and M. Schacher, "Relative Brauer groups. II', J. Reine Angew. Math. 328 (1981), 39-57.

13. M. Giudici, "Quasiprimitive permutation groups with no fixed point free elements of prime order," $J$. London Math. Soc. 67 (2003), 73-84.

14. M. Giudici, "New constructions of groups without semiregular subgroups," submitted.

15. A. Hassani, L.R. Nochefranca, and C.E. Praeger, "Two-arc transitive graphs admitting a twodimensional projective linear group," J. Group Theory 2 (1999), 335-353.

16. A.A. Ivanov and M.E. Iofinova, "Biprimitive cubic graphs, in Investigations in the Algebraic Theory of Combinatorial Objects," Kluwer, 1993, pp. 459-472.

17. A.A. Ivanov and C.E. Praeger, "On finite affine 2-arc transitive graphs," European J. Combin. (1993), 421-444.

18. D. Jordan, "Eine symmetrieeigenschaft von graphen,graphentheorie und ihre anwendungen (Stadt Wehlen, 1988)," Dresdner Reihe Forsch. 9 (1988), 17-20.

19. C.H. Li, "The finite vertex-primitive and vertex-biprimitive $s$-transitive graphs for $s \geq 4$," Trans. Amer. Math. Soc. 353 (2001), 3511-3529.

20. C.H. Li, C.E. Praeger, A. Venkatesh, and S. Zhou, "Finite locally-quasiprimitive graphs," Discrete Math. 246 (2002), 197-218.

21. C.H. Li, and Á. Seress, "Constructions of quasiprimitive two-arc transitive graphs of product action type." in A. Hulpke, R. Liebler, T. Penttila, Á. Seress (eds.), Finite Geometries, Groups and Computation, Walter de Gruyter 2006, pp. 115-124.

22. D. Marušič, "On vertex symmetric digraphs," Discrete Math. 36 (1981), 69-81.

23. D. Marušič and R. Scapellato, "Permutation groups, vertex-transitive digraphs and semiregular automorphisms," European J. Combin. 19 (1998), 707-712.

24. D. Marušič, "On 2-arc-transitivity of Cayley graphs," J. Combin. Theory Ser. B. 87 (2003), 162-196.

25. C.E. Praeger, "Imprimitive symmetric graphs," Ars Combin. 19 A (1985), 149-163.

26. C.E. Praeger, "On a reduction theorem for finite, bipartite 2-arc-transitive graphs," Australas J. Comb. 7 (1993), 21-36.

27. C.E. Praeger, "An O'Nan-Scott theorem for finite quasiprimitive permutation groups and an application to 2-arc transitive graphs," J. London Math. Soc. 47(2) (1993), 227-239.

28. C.E. Praeger, "Finite transitive permutation groups and finite vertex transitive graphs," in G. Hahn and G. Sabidussi (eds.), Graph Symmetry, NATO Advanced Science Institute Series C, Mathematical and Physical Sciences, Vol. 497, Kluwer Academic Publishing, Dordrecht, 1997, pp. 277-318.

29. C.E. Praeger, "Finite quasiprimitive graphs," in R.A. Bailey (ed.), London Mathematical Society, Lecture Note Series, Cambridge University Press, Cambridge, 1997, Vol. 241, pp. 65-85.

30. C.E. Praeger, "Finite transitive permutation groups and bipartite vertex-transitive graphs," Illinois $J$. Math. 47 (2003), 461-475.

31. C.E. Praeger and J. Saxl, "Closures of finite primitive permutation groups," Bull. London Math. Soc. 24 (1992), 251-258.

32. W.T. Tutte, "A family of cubical graphs," Proc. Cambridge Philos. Soc. 43 (1947), 459-474.

33. W.T. Tutte, "On the symmetry of cubic graphs," Canad. J. Math. 11 (1959), 621-624.

34. R. Weiss, "The nonexistence of 8-transitive graphs," Combinatorica 1 (1981), 309-311.

35. H. Wielandt, Permutation Groups Through Invariant Relations and Invariant Functions, Lecture Notes, Ohio State University, Columbus, 1969. Also publised in: Wielandt, Helmut, Mathematische Werke/Mathematical works. Vol. 1. Group theory. Walter de Gruyter \& Co., Berlin, 1994, pp. 237296. 